\title{
GENERATION OF ANALYTIC SEMIGROUPS BY STRONGLY ELLIPTIC OPERATORS UNDER GENERAL BOUNDARY CONDITIONS ${ }^{1}$
}

BY

\author{
H. BRUCE STEWART
}

\begin{abstract}
Strongly elliptic operators are shown to generate analytic semigroups of evolution operators in the topology of uniform convergence, when realized under general boundary conditions on (possibly) unbounded domains. An application to the existence and regularity of solutions to parabolic initial-boundary value problems is indicated.
\end{abstract}

Introduction. Extending the results of a previous paper [19], we propose to prove a theorem on the generation of analytic semigroups by strongly elliptic operators $A$ of order $2 m$ in the topology of uniform convergence, under more general boundary conditions. As before, there is a direct application to parabolic initial-boundary value problems: we give an existence and uniqueness theorem for such problems, using the Kato-Tanabe theory for temporally inhomogeneous evolution equations $\partial u / \partial t+A(t) u=f$. The topology of uniform convergence in the space variables yields classical solutions of this parabolic problem which are analytic in time at each space point; furthermore, the initial values are assumed in the pointwise continuous sense. Since we treat general boundary conditions, our parabolic problem is roughly comparable to the one discussed in Arima [4], where existence and uniqueness are treated (using a fundamental solution of the parabolic problem), but not analyticity.

The semigroup generation theorem for strongly elliptic operators is by now well established in the $L^{p}$ spaces. The $L^{2}$ case was treated by Browder in [5]; shortly thereafter Agmon, in [1], gave a method of proving the basic a priori estimate

$$
\|u\| \leqslant(M /|z|)\|(A+z) u\|, \quad|\arg z|<\frac{1}{2} \pi+\varepsilon,
$$

in $L^{p}$, and this method has been filled out with existence theorems and used by several authors, including Friedman [8], Higuchi [9], Lau [14], and Freeman and Schechter [7]. The work of Lau offers an attractive combination of full development and general hypotheses, so we shall borrow $L^{p}$ results from [14]. We recall that the Kato-Tanabe theorem applied in the $L^{p}$ topology gives solutions which are

Received by the editors June 25, 1979.

AMS (MOS) subject classifications (1970). Primary 35K35, 47D05.

${ }^{1}$ This work was supported in part by the U. S. Department of Energy under contract EY-76-C-02-0016. Accordingly, the U. S. Government retains a nonexclusive, royalty-free license to publish or reproduce the published form of this contribution, or allow others to do so, for U. S. Government purposes. 
analytic in $t$ in an $L^{p}$ sense; the pointwise analyticity can be deduced indirectly, but initial values are taken only in the $L^{p}$ sense.

In the topology of uniform convergence, a semigroup generation theorem also holds; this was formulated and proved by Masuda [15a] for second-order operators under Dirichlet boundary conditions, including the case of unbounded space domain. Generalization was made independently by Masuda (announced in [15b]) and Stewart [19] to higher order operators. The approach of [19], making use of the method of Agmon, seemed at once more direct than Masuda's, and easier to generalize. The extension to more general boundary conditions is the subject of the present paper.

Our results could be extended further to certain strongly elliptic operators of order $2 m$ whose coefficients have jump discontinuities across smooth nonintersecting interfaces in space; at these interfaces $2 m$ additional continuity conditions are to be specified for solutions and their derivatives. (For the a priori estimates in $L^{p}$, see e.g. Sheftel [18].) However, if the interfaces are not smooth or if they intersect each other or the boundary, the problem is more difficult. This case includes interesting applications (see Oleinik [17], Stewart [20]), but such problems are probably best solved for second-order operators using special methods.

Another topology for considering elliptic operators is the Hölder norm. Although Hölder continuity has been most useful in analyzing elliptic problems, the Hölder norm is not directly suited for a semigroup generation theorem. Recently von Wahl [22], [23] has remarked that the estimate (E) does not hold with the Hölder norm; this is because the two parts of the Hölder norm-the sup norm and the Hölder seminorm-will be associated with different powers of $|z|$. A correct estimate, albeit unsatisfactory for proving semigroup generation, can be easily obtained.

This paper comprises four sections. $§ 1$ gives the notations and the assumptions used throughout; the main results are also stated here. $\$ 2$ proves the a priori estimate involving the sup norm. In $\$ 3$ we give the existence theory needed to conclude the generation of an analytic semigroup. $\$ 4$ discusses the application to a parabolic initial-boundary value problem, which yields a solution analytic in time.

The author again wishes to express his indebtedness to Professor Tosio Kato for his kind and helpful advice.

1. Notation and main results. We shall consider a linear elliptic operator $A$ of order $2 m$ defined on an open subset $\Omega$ of $\mathbf{R}^{N}$. We include the possibility that $\Omega$ may be unbounded. Using multi-index notation we define

$$
A=A(x, D)=\sum_{|\alpha|<2 m} a_{\alpha}(x) D^{\alpha}, \quad x \in \Omega,
$$

where $D$ is the vector with $i$ th component $D_{i}=\left(\partial / \partial x_{i}\right)$, and $D^{\alpha}=$ $D_{1}^{\alpha_{1}} D_{2}^{\alpha_{2}} \cdots D_{N}^{\alpha_{N}}$. Of particular interest are the highest order terms of $A$; to separate these we introduce the symbol

$$
A^{\prime}(x, \xi)=(-1)^{m} \sum_{|\alpha|=2 m} a_{\alpha}(x) \xi^{\alpha}
$$

for any $\xi \in \mathbf{R}^{N}$, with $\xi^{\alpha}$ defined like $D^{\alpha}$. 
The operator $A$ will have complex coefficients $a_{\alpha}(x)$ subject to the following assumptions:

(A1) $A$ is uniformly strongly elliptic: there is a constant $E>0$ such that for all $x \in \Omega$ and $\xi \in \mathbf{R}^{N}, \operatorname{Re}\left(A^{\prime}(x, \xi)\right) \geqslant E|\xi|^{2 m}$.

(A2) The following smoothness assumptions hold: for $|\alpha|=2 m$ the $a_{\alpha}$ are uniformly continuous on $\Omega$ with modulus of continuity $\omega(\delta)$; for $|\alpha| \leqslant 2 m$, the $a_{\alpha}$ are continuous on $\bar{\Omega}$ and uniformly bounded by $L$.

We note that (A1) implies that the root condition is satisfied (cf. Morrey [16, p. 255]): for $x \in \partial \Omega$, and $\xi^{\prime}, \eta \in \mathbf{R}^{N}$ tangent and normal, respectively, to $\partial \Omega$ at $x$, $A^{\prime}\left(x, \xi^{\prime}+\lambda \eta\right)$ considered as a polynomial in $\lambda$ has exactly $m$ roots with positive imaginary part.

An operator $A$ will act in the open set $\Omega$ whose boundary $\partial \Omega$ possesses a certain degree of smoothness; on $\partial \Omega$ a set of $m$ boundary conditions will be imposed via linear differential boundary operators $B_{k}=\Sigma_{|\alpha|<m_{k}} b_{\alpha}^{k} D^{\alpha}$ of order $m_{k}<2 m$. We suppose the following (cf. Browder [5], Agmon,Douglis, Nirenberg [3], Lau [14]):

(B1) The boundary $\partial \Omega$ is uniformly regular of class $C^{2 m}$, that is, each point of the boundary has a coordinate transformation which locally flattens the boundary, and all the coordinate transformations $\left\{\Phi_{i}\right\}$ and their inverses have uniformly bounded and continuous derivatives of order $2 \mathrm{~m}$.

(B2) Let $B_{k}^{\prime}(x, \xi)$ be the symbol of the highest order terms of $B_{k}(x, D)$ and following the root condition define

$$
M^{+}\left(\lambda, x, \xi^{\prime}\right)=\prod_{i=1}^{m}\left(\lambda-\lambda_{i}^{+}\left(\xi^{\prime}\right)\right)
$$

where $\lambda_{i}^{+}$are the roots of $A^{\prime}\left(x, \xi^{\prime}+\lambda \eta\right)$ with positive imaginary part. We require the complementing condition, that the polynomials $B_{k}^{\prime}\left(x, \xi^{\prime}+\lambda \eta\right)$ in $\lambda$ be linearly independent modulo $M^{+}$. Furthermore, we require this condition in the following uniform sense (since $\partial \Omega$ may be unbounded): If $\Delta(x, \xi)$ is the determinant of the coefficients of the $B_{k}^{\prime}(x, \xi+\lambda \eta)\left(\bmod M^{+}\right)$, then $\Delta(x, \xi)>\Delta>0$ for all $x \in \partial \Omega$ and $\xi \in \mathbf{R}^{N}$.

(B3) The coefficients $b_{\alpha}^{k}$ of the $B_{k}$ satisfy the smoothness assumptions: all coefficients have derivatives of order $2 m-m_{k}$, denoted $D^{2 m-m_{k}} b_{\alpha}^{k}$, uniformly continuous with modulus $\omega^{\prime}(\delta)$ and uniformly bounded by $L^{\prime}$ on $\partial \Omega$.

The $L^{p}$ estimates which we shall start from use the norms

$$
\begin{aligned}
\|u\|_{j, L^{p}(\Omega)} & =\sum_{0<i<j}|[u]|_{i, L^{p}(\Omega)} \\
|[u]|_{i, L^{p}(\Omega)} & =\sum_{|\beta|=i}\left(\int_{\Omega}\left|D^{\beta} u(x)\right|^{p} d x\right)^{1 / p}
\end{aligned}
$$

on the Sobolev spaces $W^{j p}(\Omega)$. Under smoothness assumption (B1), if $j<2 m$ the space $W^{j, p}(\Omega)$ is the completion in its norm of the space $C^{\infty}(\bar{\Omega})$ of functions with infinitely many bounded derivatives on $\bar{\Omega}$. $W_{\text {loc }}^{j p}(\Omega)$ shall be all functions which are in $W^{j, p}(\Omega \cap B)$ for all closed bounded sets $B$. 
Our special interest will be the space $C_{0}(\bar{\Omega})$ of continuous functions uniformly bounded on $\bar{\Omega}$ and tending to zero as $x \rightarrow \infty$. The norm in this space is the maximum magnitude on $\bar{\Omega}$, denoted by $\|\cdot\|_{C(\bar{\Omega})}$. The space $C^{j}(\bar{\Omega})$ of functions with $j$ continuous and bounded derivatives has norm

$$
\begin{aligned}
\|u\|_{j, C(\bar{\Omega})} & =\sum_{0<i<j}|[u]|_{i, C(\bar{\Omega})}, \\
|[u]|_{i, C(\bar{\Omega})} & =\sum_{|\beta|=i}\left\|D^{\beta} u\right\|_{C(\bar{\Omega})} .
\end{aligned}
$$

Introducing a variation on notation in Lau [14], we shall use the following norms depending on a complex parameter $z$ (and also on the order $2 m$ of $A$ ):

$$
\begin{aligned}
& { }_{z}\|u\|_{j, p}=\sum_{0<i<j}|z|^{-i / 2 m}|[u]|_{i, L^{p}(\Omega)}, \\
& { }_{z}\|u\|_{j, C}=\sum_{0<i<j}|z|^{-i / 2 m}|[u]|_{i, C(\bar{\Omega})} .
\end{aligned}
$$

These powers of $|z|$ arise in developing a priori estimates for $(A+z)$. For fixed $z$ these norms are equivalent to the norms in $W^{j, p}$ and $C^{j}$ respectively. Finally we need a rather complicated seminorm which was essentially introduced by Masuda to make the sup norm estimate work. The genesis of this seminorm can be traced in $\S 2$.

$$
\begin{aligned}
{ }_{z}|[u]|_{2 m, q, z} & =\sum_{|\gamma|=2 m}|z|^{N / 2 m q}\left\|D^{\gamma} u\right\|_{q, z}, \\
\|u\|_{q, z} & =\sup _{x_{0} \in \bar{\Omega}}\|u\|_{L^{q}\left(B\left(x_{0}, r_{z}\right) \cap \Omega\right)},
\end{aligned}
$$

with $r_{z}=r_{0}|z|^{-1 / 2 m}$ and $B\left(x_{0}, r_{z}\right)$ being the open ball of radius $r_{z}$ about $x_{0}$. (The positive constant $r_{0}$ will be determined below.)

Using this notation, we state the main a priori estimate to be proved in the next section.

Theorem 1. Assume (A1)-(A2), (B1)-(B3), and let $q>N$. Then there are positive constants $\varepsilon, M, \lambda_{0}$, and $r_{0}$ such that for any $u$ in

$\mathscr{D}_{0}^{q}(A)=\left\{u \in C_{0}(\bar{\Omega})\right.$ s.t. $u \in W_{\mathrm{loc}}^{2 m, q}(\Omega), A u \in C_{0}(\bar{\Omega})$, and $B_{k} u=0$ for $\left.1<k<m\right\}$, the estimate

$$
|z|_{z}\|u\|_{2 m-1, C}+{ }_{z}|[u]|_{2 m, q, z} \leqslant M\|(A+z) u\|_{C}
$$

holds for all complex $z$ in the truncated sector $|\arg z|<\frac{1}{2} \pi+\varepsilon,|z|>\lambda$. In particular, the right side bounds $|z|\|u\|_{C}$.

REMARK. We state once and for all that the constants $\left(\varepsilon, M, \lambda_{0}\right.$, and $\left.r_{0}\right)$ depend on $q$ and on $E, L, \omega, \Delta, L^{\prime}, \omega^{\prime}$, and $\Omega$.

As previously in [19], it may be necessary to alter the space $C_{0}(\bar{\Omega})$ slightly to obtain the semigroup generation theorem. This is because the boundary conditions may include, explicitly or implicitly, the condition $u=0$ on some part or all of $\partial \Omega$; in that case any uniform limit of functions satisfying the boundary conditions 
would also vanish on the same part of $\partial \Omega$, so $\mathscr{D}_{0}^{q}(A)$ would not be dense in $C_{0}(\bar{\Omega})$. To insure a densely defined operator we introduce

$$
C_{* 0}(\bar{\Omega})=\text { completion in } C_{0}(\bar{\Omega}) \text { of } \mathscr{D}_{0}^{q}(A) \text {. }
$$

This is clearly independent of $q$, since it consists of functions in $C_{0}(\Omega)$ which vanish on $\partial \Omega$ where required by the boundary conditions. From this we set

$$
\mathscr{D}_{* 0}(A)=\left\{u \in \mathscr{D}_{0}^{q}(A), q>N \text {, with } u \text { and } A u \text { in } C_{* 0}(\bar{\Omega})\right\} \text {. }
$$

THEOREM 2. Assuming (A1)-(A2), (B1)-(B3), the operator $-A_{* 0}$ with domain $\mathscr{D}_{* 0}(A)$ generates an analytic semigroup in the space $C_{* 0}(\bar{\Omega})$ with norm $\|\cdot\|_{C}$.

Finally, our application is the following temporally inhomogeneous parabolic initial-boundary value problem.

THEOREM 3. Consider the parabolic problem

$$
\begin{aligned}
\partial u / \partial t+A(t) u(t, x) & =f(t, x), \quad t>0, x \in \Omega, \\
u(0, x)=u_{0}(x), \quad x \in \Omega, & \quad \quad \quad \quad 1 \leqslant k \leqslant m, x \in \partial \Omega,
\end{aligned}
$$

where $A(t), B_{k}(t)$ satisfy (A1)-(A2), (B1)-(B3) for $\Omega$, uniformly for $t>0 ; u_{0}$ and $f(t)$ are in $C_{* 0}(\bar{\Omega})$; and $f(t), \partial a_{\alpha} / \partial t$, and $\partial\left(D^{2 m-m_{k}} b_{\alpha}^{k}\right) / \partial t$ satisfy a Hölder condition in $t$ uniformly on $\bar{\Omega}$, e.g. $\|f(t)-f(s)\|_{C} \leqslant|t-s|^{\nu}$. Then (1.2) has a unique solution as an abstract evolution equation in $C_{* 0}(\bar{\Omega})$, with the following concrete properties:

(i) $u(t, x)$ is uniformly continuous in $[0, T] \times \bar{\Omega}$, and the initial values $u_{0}(x)$ are taken on continuously.

(ii) $\partial u / \partial t$ exists and is continuous in $(0, T] \times \bar{\Omega}$.

(iii) For $t>0, u(t, x)$ has $x$-derivatives up to order $2 m-1$ in the continuous sense and of order $2 m$ in the $L^{q}$ sense, $q>N$, and the boundary conditions are satisfied continuously.

(iv) If $f, a_{\alpha}$, and $D^{2 m-m_{k}} b_{\alpha}^{k}$ are analytic in t, uniformly in $\bar{\Omega}$, in a small sector in the complex plane about $[0, T]$, then so is $u(t, x)$ for a slightly smaller sector about $(0, T]$.

(v) If in addition to (iv) $f, a_{\alpha}$, and $D^{2 m-m_{k}} b_{\alpha}^{k}$ are Hölder continuous in $x$, then $u(t, x)$ has Hölder continuous $x$-derivatives of order $2 m$ and satisfies the differential equation in the continuous sense.

2. Estimate. The proof of estimate (1.1) of Theorem 1 goes much as in [19] under Dirichlet boundary conditions. We start by establishing $L^{p}$ estimates for $(A+z)$ in the norm ${ }_{z}\|\cdot\|_{2 m, p}$ in the simplified case of homogeneous constant coefficient operators on a whole or half-space. The idea for this step is due to Agmon [1]. From the $L^{p}$ estimates we deduce estimates involving ${ }_{z}\|\cdot\|_{2 m-1, C}$ and $\|\left.[\cdot]\right|_{2 m, L^{q}}$ via the Sobolev lemma and a dilation of coordinates to obtain the correct dependence on $|z|$. All this is still for constant coefficients. The general case requires the usual sort of localization argument, but with a special choice of constants.

The present derivation differs in one aspect from [19]: it will be necessary to carry along certain norms of boundary values. Following Agmon, Douglis, and 
Nirenberg $\left[3\right.$, p. 648], if $\phi_{k}$ is the value on $\partial \Omega$ of a function $v \in W^{j p}(\Omega)$, then we define

$$
\left|\left[\phi_{k}\right]\right|_{j-1 / p}=\inf |[v]|_{j, L^{p}(\Omega)}
$$

where the infimum is taken over all such $v$ having boundary value $\phi_{k}$. For boundary values $\phi=\left(\phi_{1}, \ldots, \phi_{m}\right), \phi_{k}=B_{k} u$, we define

$$
|[\phi]|_{2 m-1 / p}=\sum_{k=1}^{m}|[\phi]|_{2 m-m_{k}-1 / p}
$$

We start with a strongly elliptic operator $A^{\#}$ having constant coefficients of order $2 m$ only, satisfying (A1) and the bound of (A2). The domain will be either $\mathbf{R}^{N}$, or the half-space $\mathbf{R}_{+}^{N}$ with boundary conditions $B_{k}^{\#}$ at $x_{N}=0$. Each boundary condition will also have constant coefficients of highest order $m_{k}$ only, satisfying (B2) and the bound of (B3). For these constant coefficient cases we have the following $L^{p}$ estimates.

LEMMA 1. Let $A^{\#}$ and $\left\{B_{k}^{\sharp}\right\}$ be the constant coefficient operators above. For $1<p<\infty$, there are positive constants $\varepsilon, M$, such that for all $u \in W^{2 m, p}\left(\mathbf{R}_{+}^{N}\right)$ with compact support in $B(0,1)$,

$$
|z|_{z}\|u\|_{2 m, p} \leqslant M\left\{\left\|\left(A^{\#}+z\right) u\right\|_{L^{p}}+\left|\left[B^{\sharp} u\right]\right|_{2 m-1 / p}\right\}
$$

holds for $\mathbf{R}_{+}^{N}$ for $|\arg z| \leqslant \frac{1}{2} \pi+\varepsilon$. The same estimate holds on $\mathbf{R}^{N}$ without the boundary values.

Proof. The idea, due to Agmon [1], consists of defining a new operator $T$ in $\mathbf{R}^{N+1}$ by

$$
T=A^{\#}+(-1)^{m} e^{i \theta}(\partial / \partial t)^{2 m}
$$

which will be elliptic for $|\theta|<\frac{1}{2} \pi+\varepsilon$, where $\varepsilon>0$ depends on $E$ and $L$. The a priori estimates of Agmon, Douglis, and Nirenberg [3] hold for $T$, and by choosing suitable functions on the cylinder $\Omega \times \mathbf{R}$ in the domain of $T$ one deduces (2.1). See [19] for Dirichlet boundary conditions, and Lau [14] for the general case. (We use seminorms of $B^{\sharp} u$ where Lau uses norms; but our statement is clearly permitted by the homogeneity of orders of $A^{\#}$ and of the $B_{k}^{\sharp}$. Cf. the proof of Theorem 14.1 in [3].)

We now pass to the local estimates involving $C$ norms.

Lemma 2. Let $q>N$ and set $\theta=N / 2 m q$. Under the same conditions as Lemma 1 we have the estimate

$$
|z|_{z}\|u\|_{2 m-1, C}+|z|^{\theta}|[u]|_{2 m, L^{q}}<M|z|^{\theta}\left\{\left\|\left(A^{\#}+z\right) u\right\|_{L^{q}}+\left|\left[B^{\#} u\right]\right|_{2 m-1 / q}\right\} .
$$

Proof. First fix $z=z_{0},\left|z_{0}\right|=1$, (but allowing the argument to vary). If $q>N$, the Sobolev lemma gives

$$
z_{0}\|u\|_{2 m-1, C}<K_{z_{0}}\|u\|_{2 m, L^{a}},
$$

which leads to (2.2) for $z_{0}$. To regain the dependence on $|z|$ we dilate the spatial coordinates by a factor depending on $|z|$. Define $\rho=|z|^{-1 / 2 m}, y=\rho^{-1} x$, and 
$u^{\prime}(y)=\rho^{-2 m} u(x)$. Derivatives and integral norms of $u^{\prime}$ are to be understood consistently in the variable $y$. If $u \in W^{2 m, q}\left(\mathbf{R}_{+}^{N}\right)$ and $B_{k}^{\sharp} u=\phi_{k}(x)$ on $x_{N}=0$, $1<k \leqslant m$, then $u^{\prime} \in W^{2 m, q}\left(\mathbf{R}_{+}^{N}\right)$ and $B_{k}^{\sharp} u^{\prime}=\rho^{2 m-m_{k}} \phi_{k}(y)$. Furthermore $\left(A^{\sharp}+z\right) u$ $=\left(A^{\#}+\rho^{2 m} z\right) u^{\prime}$. By including the effect of change of variable of integration, we find

$$
\begin{aligned}
& \left\|\left(A^{\#}+z\right) u\right\|_{L^{q}}=\rho^{N / q} \|\left(A^{\#}+\rho^{\left.2 m_{z} z\right) u^{\prime} \|_{L^{q}},}\right. \\
& \|\left.\left[B^{\sharp} u\right]\right|_{2 m-1 / q}=\rho^{2 m-m_{k}-\left(2 m-m_{k}\right)+N / q}\left|\left[B^{\sharp} u^{\prime}\right]\right|_{2 m-1 / q^{\circ}}
\end{aligned}
$$

Now we set $z_{0}=\rho^{2 m} z$; substituting into (2.2), we pass from $u^{\prime}$ and $\left|z_{0}\right|=1$ to the general case of $u$ and $z$.

Proof of Theorem 1. We proceed as follows. First the constant coefficient estimate (2.2) is extended to variable coefficient operators whose leading coefficients vary only slightly. By a smooth change of coordinates this leads to local estimates in a general domain $\Omega$ with smooth boundary. The local estimates are combined by taking their supremum. Finally a careful choice of localization parameters will adjust the $(A+z)$ term in the inequality to give (1.1).

Let $A, B$ be defined on $G=B(0, \delta) \cap \mathbf{R}_{+}^{N}$ with variable coefficients satisfying (A1), (A2), (B2), (B3). Let $u \in W^{2 m, q}\left(\mathbf{R}_{+}^{N}\right)$ have support in $\bar{G}$ and satisfy the boundary conditions $B u=\phi$ at $x_{N}=0$. Choose $A^{\sharp}=A^{\prime}(0, D)$ and $B^{\sharp}=B^{\prime}(0, D)$, constant coefficient operators consisting of the highest order terms of $A, B$ evaluated at $x=0$. We may apply Lemma 2 . If we write

$$
A^{\#}=A-\sum_{|\alpha|=2 m}\left(a_{\alpha}(x)-a_{\alpha}(0)\right) D^{\alpha}-\sum_{|\alpha|<2 m} a_{\alpha}(x) D^{\alpha}
$$

and similarly for $B^{\sharp}$, we get from (2.2)

$$
\begin{aligned}
& |z|_{z}\|u\|_{2 m-1, C}+|z|^{\theta}|[u]|_{2 m, L^{q}} \\
& \leqslant M|z|^{\theta}\left\{\|(A+z) u\|_{L^{q}}+\omega|[u]|_{2 m, L^{q}}+L\|u\|_{2 m-1, L^{q}}\right. \\
& \left.\quad+|[B u]|_{2 m-1 / q}+m \omega^{\prime}|[u]|_{2 m, L^{q}}+m L^{\prime}\|u\|_{2 m-1, L^{q}}\right\}
\end{aligned}
$$

where by assumption (A2), $\omega=\omega(\delta)=\sup \left|a_{\alpha}(x)-a_{\alpha}(0)\right|$, and $\omega^{\prime}$ similarly bounds the variation of highest order coefficients of $B ; L$ and $L^{\prime}$ are the coefficient bounds. We have used the fact that

$$
\left|\left[D^{\alpha} u\right]\right|_{2 m-m_{k}-1 / q}<\|u\|_{|\alpha|+2 m-m_{k}, L^{q}(G)}
$$

which follows from the definition of the boundary norms. As usual we subtract the extra terms on the right of (2.3) from both sides: for the $\omega, \omega^{\prime}$ terms by choosing $0<\delta<\delta_{0}$ with $\delta_{0}$ small enough, for the $L, L^{\prime}$ terms by choosing $|z|>\lambda_{0}$ with $\lambda_{0}$ large enough. In the latter instance, we note that

and

$$
\|u\|_{j, L^{q}(G)} \leqslant(\operatorname{meas} G)^{1 / q}\|u\|_{j, C(G)}<K \delta^{N / q}\|u\|_{j, C(G)}
$$

$$
|z|_{z}\|u\|_{2 m-1, C} \geqslant|z|^{1 / 2 m}\|u\|_{2 m-1, C}
$$


for $|z| \geqslant 1$, so we need

$$
\frac{1}{2} \lambda_{0}^{1 / 2 m} \geqslant M\left(L+m L^{\prime}\right) K \delta_{0}^{N / q} \lambda_{0}^{\theta}
$$

which is possible because with $q>N, \theta<1 / 2 \mathrm{~m}$. We thus have estimate (2.2) for slightly varying coefficients. The hypothesis that the function $u$ has compact support is now essential, as is the lower bound for $|z|$.

Now consider a point $x_{0}$ on the smooth boundary $\partial \Omega$ of $\Omega$. By assumption (B1), a smooth coordinate transformation $\Phi$ maps $B\left(x_{0}, r_{0}\right) \cap \Omega$ into $B(0, \delta) \cap \mathbf{R}_{+}^{N}$ and flattens a part of $\partial \Omega$ into the plane $y_{N}=0$. It is well known that such a change of variable preserves the properties (A1), (A2), (B2), and (B3) of $A$ and $B$. Thus one verifies that (2.2) maps into a local estimate for $A, B$ in $\Omega$.

Of course $u$ need not have compact support in $B\left(x_{0}, r_{0}\right)$, so instead of $u$ we shall consider $\phi_{0} u$, where $\phi_{0}$ is a localizing function. Let $\phi_{0}=\phi\left(\left(x-x_{0}\right) / r\right)$, where $\phi$ is infinitely differentiable with support in $B(0,1)$ and identically 1 on $B\left(0, \frac{1}{2}\right)$. The choice of $r\left(<r_{0}\right)$ will be made later. Let $G^{\prime}=B\left(x_{0}, r\right) \cap \Omega$ and $G^{\prime \prime}=B\left(x_{0}, \frac{1}{2} r\right) \cap$ $\Omega$. (Interior points are treated in similar fashion.) We have

$$
\begin{aligned}
|z|_{z}\|u\|_{2 m-1, C\left(G^{\prime \prime}\right)}+ & |z|^{\theta}|[u]|_{2 m, L^{q}\left(G^{\prime \prime}\right)} \leqslant|z|_{z}\left\|\phi_{0} u\right\|_{2 m-1, C\left(G^{\prime}\right)}+|z|^{\theta}\left|\left[\phi_{0} u\right]\right|_{2 m, L^{q}\left(G^{\prime}\right)} \\
& \leqslant M|z|^{\theta}\left\{\left\|(A+z) \phi_{0} u\right\|_{L^{q}\left(G^{\prime}\right)}+\left|\left[B\left(\phi_{0} u\right)\right]\right|_{2 m-1 / q}\right\} .
\end{aligned}
$$

Expanding $A\left(\phi_{0} u\right)=\phi_{0} A u+\cdots$ (where the dots represent terms with lower order derivative of $u$ multiplied by derivatives of $\left.\phi_{0}\right)$, and similarly for $B\left(\phi_{0} u\right)$, and noting that $D^{\alpha} \phi_{0} \leqslant K r^{-|\alpha|}$, we bound the expression displayed above by

$$
\begin{aligned}
& \leqslant M|z|^{\theta}\left\{\|(A+z) u\|_{L^{q}\left(G^{\prime}\right)}+|[B u]|_{2 m-1 / q}+r^{-1}\|u\|_{2 m-1, L^{q}\left(G^{\prime}\right)}\right\} \\
& \leqslant M|z|^{\theta}\left\{r^{N / q}\|(A+z) u\|_{C\left(G^{\prime}\right)}+r^{-1+N / q}\|u\|_{2 m-1, C\left(G^{\prime}\right)}\right\},
\end{aligned}
$$

assuming that $u \in \mathscr{D}_{0}^{q}(A)$. Now we take the supremum over all $x_{0}$ in $\Omega$ and get

$$
\begin{aligned}
|z|_{z}\|u\|_{2 m-1, C(\Omega)}+|z|^{\theta} \sup _{x_{0} \in \Omega}|[u]|_{2 m, L^{q}\left(B\left(x_{0}, r / 2\right) \cap \Omega\right)} & \\
& \leqslant M|z|^{\theta}\left\{r^{N / q}\|(A+z) u\|_{C(\Omega)}+r^{-1+N / q}\|u\|_{2 m-1, C(\Omega)}\right\} .
\end{aligned}
$$

We now choose constants (this was incorrectly explained in [19]). Let $\lambda_{0}$ and $r_{0}$ be as above (to subtract the $\omega$ and $L$ terms from (2.3)). Set $r=K r_{0}|z|^{-1 / 2 m}$; the right side becomes

$$
\leqslant M K^{N / q}\|(A+z) u\|_{C(\Omega)}+M K^{-1+N / q}|z|^{1 / 2 m}\|u\|_{2 m-1, C(\Omega)}
$$

after absorbing $r_{0}$ into $M$. Now choose $K$ large enough to subtract the last term from both sides (the exponent $-1+N / q$ is negative). This leaves the desired inequality. (It may be necessary to increase $\lambda_{0}$ further to guarantee $r<r_{0}$.)

REMARK. The proof requires a $z$-dependent localization argument, even in the simplest case $A=A^{\sharp}, \Omega=\mathbf{R}^{N}$. If we try to pass from Lemma 2 directly to (1.1), we find that the support of $u$ would be restricted to a ball of radius inversely proportional to $|z|^{1 / 2 m}$.

3. Existence. Theorem 1 can be translated into a semigroup generation theorem provided the existence of solutions of $(A+z) u=f$ can be shown. In that case 
$(A+z)$ will have a right inverse, and the necessary resolvent estimate is an easy consequence of (1.1).

We define the realization $A_{0}^{q}$ of $A$ to be the operator with domain $\mathscr{Q}_{0}^{q}(A) \subset$ $C_{0}(\bar{\Omega})$ and range in $C_{0}(\bar{\Omega})$ given by $A_{0}^{q} u=A u$ for each $u$ in the domain. For this realization we have:

Theorem 4. Let (A1)-(A2), (B1)-(B3) hold, and suppose $q>N$. Then $A_{0}^{q}$ is a closed operator independent of $q$, which we call $A_{0}$. For $|z|>\lambda_{0}$ and $|\arg z|<\frac{1}{2} \pi+\varepsilon$, $A_{0}+z$ has a bounded right inverse on $C_{0}(\bar{\Omega})$.

Proof. That $A_{0}^{q}$ is closed follows from the a priori estimate of Theorem 1, to which the definition of $\mathscr{D}_{0}^{q}(A)$ has been adapted.

To show that $A_{0}^{q}+z$ has dense range, consider $f \in C^{\infty}(\bar{\Omega})$ having compact support. Then in particular $f \in L^{q}(\Omega)$, and according to Theorem 2.7 of Lau [14], the realization of $A$ in $W^{2 m, q}(\Omega)$ has a right inverse, i.e. the equation $(A+z) u=f$ has a solution $u \in W^{2 m, q}(\Omega)$ satisfying $B u=0$. It follows that $u \in C^{2 m-1}(\bar{\Omega})$ and of course $u \in W_{\mathrm{loc}}^{2 m, q}(\Omega)$. Since $u \in C^{1}(\bar{\Omega}) \cap L^{q}(\Omega)$, it must be that in the case of unbounded $\Omega, u(x) \rightarrow 0$ as $|x| \rightarrow \infty$. Thus $u$ is in $C_{0}(\bar{\Omega})$. Since $f$ is also in $C_{0}(\bar{\Omega})$, it follows from $(A+z) u=f$ that $A u \in C_{0}(\bar{\Omega})$ as well. This shows that $u \in \mathscr{D}_{0}^{g}(A)$.

Thus $A_{0}^{q}+z$ has dense range in $C_{0}(\bar{\Omega})$ for $z$ in a sector $S_{q}$ of the complex plane. (For the moment $\lambda_{0}$ depends on $q$.) By the Closed Range Theorem, $A_{0}^{q}+z$ maps onto $C_{0}(\bar{\Omega})$. Let $q^{\prime}>q$. For $z \in S_{q} \cap S_{q^{\prime}}$, it is clear from $\operatorname{range}\left(A_{0}^{q}+z\right)=$ range $\left(A_{0}^{q^{\prime}}+z\right)=C_{0}(\bar{\Omega})$ that we must have $\mathscr{D}_{0}^{q}(A)=\mathscr{D}_{0}^{q^{\prime}}(A)$.

Thus we define $A_{0}$, independent of $q$, and from Theorem 1 the inverse of $A_{0}+z$ is bounded by a constant over $|z|$.

We have almost shown that $-A_{0}$ generates an analytic semigroup (cf. Kato [10, Chapter IX]). The operator $A_{0}$ lacks only a domain dense in $C_{0}(\bar{\Omega})$. To obtain this, we call on the space $C_{* 0}(\bar{\Omega})$ as defined in $\S 1$.

Proof of Theorem 2. To show that the realization $A_{* 0}$ of $A$ with domain $\mathscr{D}_{* 0}(A)$ is densely defined, consider $C_{* c}^{\infty}(\bar{\Omega})$, the set of infinitely differentiable functions with compact support in $\bar{\Omega}-\Gamma$, where $\Gamma$ is the closed subset of $\partial \Omega$ where $B u=0$ implies $u=0$. This set of functions is dense in $C_{* 0}(\bar{\Omega})$, and for $u \in$ $C_{* c}^{\infty}(\bar{\Omega})$, we see that $A u \in C_{* 0}(\bar{\Omega})$. (Here we use the continuity of the lower order coefficients of $A$ for the first time.)

One sees readily that $A_{* 0}$ is closed and that $A_{* 0}+z$ has bounded inverse on $C_{* 0}(\bar{\Omega})$. Thus the hypotheses of the analytic semigroup generation theorem are satisfied by $-A_{* 0}$.

4. Application. We have now proved Theorem 2, that a strongly elliptic operator with general boundary conditions generates an analytic semigroup in the topology of uniform convergence. Such a semigroup $U(t)$ gives uniquely determined solutions of the abstract evolution problem $\partial u / \partial t+A u=0, u(0)=u_{0}$. More specifically, this corresponds to a temporally homogeneous parabolic initial-boundary value problem. Since the topology is uniform convergence on the space domain $\Omega$, we find that the solution $u(t, x)$ is analytic on $t$ for each $x$, and takes on the initial values $u_{0}(x)$ continuously. 
By calling on the Kato-Tanabe theory of evolution equations with time-dependent domain, our results also lead to information about temporally inhomogeneous parabolic problems. Rather than give proofs in detail, we will only sketch the main lines along which one can proceed.

We shall refer to another realization of $A, A_{q}$, which is close to $A_{0}^{q}$, but with domain independent of $A$. Let $\mathcal{X}_{q}$ be the Banach space obtained by completing $L^{q}(\Omega)$ (or equivalently $C_{0}(\bar{\Omega})$ ) under our norm $\|\cdot\|_{q, z}$. Furthermore, let $\|\cdot\|_{2 m-1 / q, z}$ be the boundary norm based on $\|\cdot\|_{q, z}$, and $\mathscr{Y}_{q}$ the completion of the boundary space $W^{2 m-1 / q, q}(\partial \Omega)$ (cf. ADN [3, p. 699], Lau [14, p. 43]). We also introduce $L u$ to stand for $\left(A u, B_{1} u, \ldots, B_{m} u\right)$.

TheOrem 5. Let (A1)-(A2), (B1)-(B3) be satisfied, and choose $q>N$. The operator $L_{q}$ with domain

$$
\mathscr{D}_{q}=\left\{u \in C_{0}(\bar{\Omega}) \text { with } D^{\alpha} u \in \mathcal{X}_{q} \text { for }|\alpha|<2 m\right\}
$$

is a closed mapping from $C_{0}(\bar{\Omega})$ into $\mathcal{X}_{q} \times \mathcal{Y}_{q}$ for which

$$
|z|\|u\|_{2 m-1, C}+{ }_{z}|[u]|_{2 m, q, z} \leqslant M|z|^{\theta}\left\{\|(A+z) u\|_{q, z}+\|B u\|_{2 m-1 / q}\right\}
$$

for $|z| \geqslant \lambda_{0},|\arg z| \leqslant \frac{1}{2} \pi+\varepsilon$, and for all $u \in \mathscr{D}_{q}$. The operator

$$
L_{q}+z=\left((A+z) u, B_{1} u, \ldots, B_{m} u\right)
$$

has right inverse on $\mathcal{X}_{q} \times \mathcal{Y}_{q}$.

Proof. The result is essentially contained in the proofs of Theorems 1 and 4. In the former, we simply refrain from introducing $\|\cdot\|_{c}$ on the right of the a priori estimate. To prove the rest we note that if $u \in \mathscr{D}_{q}, L_{q} u$ is indeed in $\mathscr{X}_{q} \times \mathscr{Y}_{q}$, and (4.1) for any fixed $z$ shows that $L_{q}$ is closed. The invertibility of $L_{q}+z$ follows as in Theorem 4 from results of Lau in $L^{q}(\Omega)$.

RemarK. We assume below that $A+z$ is invertible for $z=0$, which is no loss of generality for parabolic problems; we simply replace $A$ by $A+\lambda$ and solve $\partial v / \partial t+(A+\lambda) v=\exp (-\lambda t) f$ with $v=\exp (-\lambda t) u$.

Proof of TheOREM 3. We restrict ourselves to an outline. The hypotheses of the Kato-Tanabe theorem (cf. Kato [11, Theorem 3.1], or Kato and Tanabe [12]) are: (1) that $-A(t)$ for fixed $t$ generate an analytic semigroup, uniformly in $t$; (2) that $(\partial / \partial t)(A(t)+z)^{-1}$ exist in a sector $|\arg z|<\frac{1}{2} \pi+\varepsilon$ and be bounded in norm by a constant over $|z|$; (3) that $(\partial / \partial t)(A(t))^{-1}$ be Hölder continuous in $t$ in operator norm.

We know that hypothesis (1) is satisfied by the realizations $-A_{* 0}(t)$ in $C_{* 0}(\bar{\Omega})$. To check (2), we first let

$$
[A(t+h)+z] u(t+h)=f(t)
$$

and apply $[L(t)+z]^{-1}[L(t)+z]$ to the difference quotient $h^{-1}[u(t+h)-u(t)]$ to see that formally

$$
-\partial / \partial t(A(t)+z)^{-1} \supset(L(t)+z)^{-1} L_{t}(t)(A(t)+z)^{-1}
$$

where $L_{t}(t)$ is formed with coefficients $\partial a_{\alpha} / \partial t$ and $\partial b_{\alpha}^{k} / \partial t$. We consider the three operators on the right of (4.2), in their sequence of application, realized in the 
spaces

$$
C_{* 0}(\bar{\Omega}) \rightarrow \mathscr{D}_{q} \rightarrow \mathcal{X}_{q} \times \mathscr{Y}_{q} \rightarrow C_{* 0}(\bar{\Omega})
$$

Making use of Theorem 5, one can complete (4.2) to give an operator on all of $C_{* 0}(\bar{\Omega})$. Using the sequence of norms

$$
\|\cdot\|_{C} \rightarrow_{z}|[\cdot]|_{2 m, q, z} \rightarrow|z|^{\theta}\left(\|\cdot\|_{q, z}+\|\cdot\|_{2 m-1 / q, z}\right) \rightarrow|z|\|\cdot\|_{C}
$$

we obtain the bound required by (2).

The same relation (4.2), which is now an equality, is used to verify hypothesis (3). Because of our assumptions on the coefficients, $L_{t}(t)$ is clearly $t$-Hölder continuous in the appropriate norms in (4.3). For $L(t)^{-1}$, choose $v(t)$ a solution of $A(t) v(t)=$ $f, B(t) v(t)=g$, and similarly for $v(s)$ at $s$. Putting $|v(t)-v(s)|$ in the estimate of Theorem 5, we can then deduce the Hölder continuity in $t$ of $L(t)^{-1}$. The hypotheses of the Kato-Tanabe theorem are satisfied.

Conclusions (i)-(iv) of Theorem 3 are translations of the Kato-Tanabe conclusions into concrete form in the topology of uniform convergence. Conclusion (iv) that $u(t, x)$ has Hölder continuous $x$-derivatives of order $2 m$ if the coefficients $a_{\alpha}(t, x)$ are Hölder continuous in $x$, is proved as in [15a] (see [19]) by extending $u(t, x)$ to complex $t=\xi+i \eta$ and treating the elliptic equation

$$
\left[\left(-\Delta_{\xi \eta}\right)^{m}+A+(\partial / \partial \xi)\right] u(\xi+i \eta, x)=f(\xi+i \eta, x)
$$

as an elliptic regularity problem.

REMARK. By using another result of Kato and Tanabe, we may perturb $L(t)$ by any lower-order operator whose coefficients are Hölder continuous in $t$ uniformly in $x$ (cf. Lau [14, p. 46]). In effect this means that only the highest order coefficients need have Hölder continuous $t$-derivatives.

In order to prove the continuous differentiability in $x$ up to order $2 m$, we imposed extra smoothness assumptions on the coefficients. The conditions of Hölder continuity in $x$ are expected for this type of problem. However we have also imposed analyticity in $t$, which allows us to stay in the realm of elliptic theory. As remarked in the introduction, the holomorphic semigroup estimate is not valid in Hölder norms, so that approach to regularity is apparently not open. Noting this, von Wahl [22], [23] has considered fractional powers of elliptic operators in Hölder spaces, and proved certain regularity theorems for a linear parabolic initialboundary value problem like (1.2) with Dirichlet boundary conditions, based on elliptic theory. Unfortunately, as noted in [23], this approach also encounters some difficulty with the derivatives of order $2 \mathrm{~m}$. With parabolic methods Arima [4] has proved full $2 m$ order differentiability in $x$ for solutions of (1.2), under smoothness assumptions weaker than ours.

\section{REFERENCES}

1. S. Agmon, On the eigenfunctions and on the eigenvalues of general elliptic boundary value problems, Comm. Pure Appl. Math. 15 (1962), 119-147.

2. ___ Lectures on elliptic boundary value problems, Van Nostrand, Princeton, N. J., 1965.

3. S. Agmon, A. Douglis and L. Nirenberg, Estimates near the boundary for solutions of elliptic partial differential equations satisfying general boundary conditions. I, Comm. Pure Appl. Math. 12 (1959), 623-727. 
4. R. Arima, On general boundary value problems for parabolic equations, J. Math. Kyoto Univ. 4 (1964), 207-243.

5. F. E. Browder, On the spectral theory of elliptic differential operators. I, Math. Ann. 142 (1960/61), 22-130.

6. S. D. Eidel'man, Parabolic systems, "Nauka", Moscow, 1964; English transl., Noordhoff, Groningen; North-Holland, Amsterdam, 1969.

7. R. S. Freeman and M. Schechter, On the existence, uniqueness and regularity of solutions to general elliptic boundary value problems, J. Differential Equations 15 (1974), 213-246.

8. A. Friedman, Partial differential equations, Holt, Rinehart and Winston, New York, 1969.

9. Y. Higuchi, A priori estimates and existence theorems on elliptic boundary value problems for unbounded domains, Osaka J. Math. 5 (1968), 103-135.

10. T. Kato, Perturbation theory for linear operators, Springer-Verlag, New York, 1966.

11. Semigroups and temporally inhomogeneous evolution equations, C.I.M.E. lecture notes, Varenna, 1963.

12. T. Kato and H. Tanabe, On the abstract evolution equations, Osaka J. Math. 14 (1962), 107-133.

13. $\longrightarrow$ On the analyticity of solution of evolution equations, Osaka J. Math. 4 (1967), 1-4.

14. R. L. Lau, Elliptic equations with parameter and applications to parabolic problems, Ph.D. thesis, Yale University, 1967.

15a. K. Masuda, Manuscript for seminar at Kyoto Univ., 1970.

15b. __ Unpublished manuscript, 1972.

16. C. B. Morrey, Jr., Multiple integrals in the calculus of variations, Springer-Verlag, New York, 1966.

17. O. A. Oleinik, Boundary value problems for equations of elliptic and parabolic type with discontinuous coefficients, Izv. Akad. Nauk SSSR Ser. Mat. 25 (1961), 3-20. (Russian)

18. Z. G. Sheftel, Estimates in $L_{p}$ of solutions of elliptic equations with discontinuous coefficients and satisfying general boundary conditions and conjugacy conditions, Dokl. Akad. Nauk SSSR 149 (1963), 48-51 = Soviet Math. Dokl. 4 (1963), 321-324.

19. H. B. Stewart, Generation of analytic semigroups by strongly elliptic operators, Trans. Amer. Math. Soc. 199 (1974), 141-162.

20. __ Spectral theory of heterogeneous diffusion systems, J. Math. Anal. Appl. 54 (1976), 59-78.

21. H. Tanabe, On Green's functions of elliptic and parabolic boundary value problems, Proc. Japan Acad. 48 (1972), 709-711.

22. W. von Wahl, Gebrochene Potenzen eines elliptischen Operators und parabolische Differentialgleichungen in Räumen hölderstetiger Funktionen, Nachr. Akad. Wiss. Göttingen Math.-Phys. K1. II (1972), 231-258.

23. __ Einige Bemerkungen zu meiner Arbeit "Gebrochene Potenzen eines elliptischen Operators und parabolische Differentialgleichungen in Räumen hölderstetiger Funktionen," Manuscripta Math. 11 (1974), 199-201.

Applied Mathematics Department, Brookhaven National Laboratory, Upton, New York 11973 